\title{
Effect of Guide Vanes on Flow and Vibroacoustic in an Axial-Flow Pump
}

\author{
Guoping Li, ${ }^{1}$ Eryun Chen $\mathbb{D}^{2,3}$ Ailing Yang $\mathbb{D}^{2},{ }^{2}$ Zhibin Xie, $^{2}$ and Gaiping Zhao ${ }^{4}$ \\ ${ }^{1}$ Shanghai Marine Equipment Research Institute, Shanghai 200031, China \\ ${ }^{2}$ School of Energy and Power Engineering, University of Shanghai for Science and Technology, Shanghai 200093, China \\ ${ }^{3}$ Shanghai Key Laboratory of Multiphase Flow and Heat Transfer in Power Engineering, Shanghai 200093, China \\ ${ }^{4}$ School of Medical Instruments and Food Engineering, University of Shanghai for Science and Technology, Shanghai 200093, China
}

Correspondence should be addressed to Eryun Chen; cheneryun@usst.edu.cn

Received 20 March 2018; Revised 9 September 2018; Accepted 25 September 2018; Published 30 October 2018

Academic Editor: Nicolas Gourdain

Copyright (C) 2018 Guoping Li et al. This is an open access article distributed under the Creative Commons Attribution License, which permits unrestricted use, distribution, and reproduction in any medium, provided the original work is properly cited.

An effect of guide-vane numbers on pressure fluctuations and structural vibroacoustics induced by unsteady flow is performed by a hybrid numerical method. A 3D flow field is simulated in axial-flow pump with four impeller blades, in which three diffuser models with 5, 7, and 9 vanes are devised to match, respectively. A full scale structural vibroacoustics coupled model is solved using LMS acoustics software. The results show that the blade-passing frequency (BPF) is dominated frequency of the vibration acceleration of pump, which is consistent with frequency spectral characteristics of pressure pulsation. The unsteady pressure fluctuating becomes strong as the flow discharge decreases from $1.0 \mathrm{Qv}$ to $0.6 \mathrm{Qv}$, the circumferential unsteady behavior of which is more severe due to flow nonuniformity induced by the suction elbow at partial operation. Generally, the pressure fluctuating increases slightly when the flow discharge increases from 1.0Qv to $1.3 \mathrm{Qv}$. Moreover, pressure fluctuations amplitude on the pump with 9-vane diffuser is small relative to other two models and the vibrating accelerating and radiation sound field at BPF are also slight relatively, which indicates that appropriate guide-vane numbers contribute to suppress pressure fluctuations and vibroacoustics in axial-flow pump. The conclusions in the present paper can provide theoretical guidance for low vibration pump design.

\section{Introduction}

Axial-flow pumps are usually selected for pumping large flow rate against relatively low heads [1] and widely used in the military, marine, agricultural, and biomedical fields. It is thus expected that the fluid transportation is accompanied by little vibronoise. The guide vanes mounted in the downstream of the impeller can improve the hydrodynamic performance of the pump by transforming the kinetic energy into pressure energy. But the rotor-stator mutual interaction can induce unsteady flow forces on the pump, causing vibration and noise [2], leading to structural fatigue failures. Therefore, the investigation of rotor-stator interaction [3-5] effects on pressure fluctuation and vibration in the axial-flow pump is of continuous interest.

The interaction between rotor and stator is always a hot issue because it has important influence on the flow structure, performance, and operation stability of the turbomachinery.
Experiment study of the flow field structures between the rotors and stators was employed by Zhang [6]. Zierke $[7,8]$ studied the tip clearance flow field structures using flow visualization experiment technology, giving examples of measured physical phenomena such as endwall boundary layers, separation regions, wakes, and secondary vortical structures. With the rapid development of computers and computational method, the numerical simulation makes even more attractive technique to research the inner flow in pumps. Several numerical studies have been reported in the technical literature on rotor and stator interaction [9-11]. For example, Javadi [9] investigated the precessing helical vortex in a conical diffuser, with rotor-stator interaction, which showed the rotor-stator interaction is necessary to reproduce the physics of the helical vortex in the draft tube. Liu [10] presents an analytical investigation of stator lean on rotor-stator interaction noise in the compressor, which indicated that stator-leaned positive has better effect on noise 


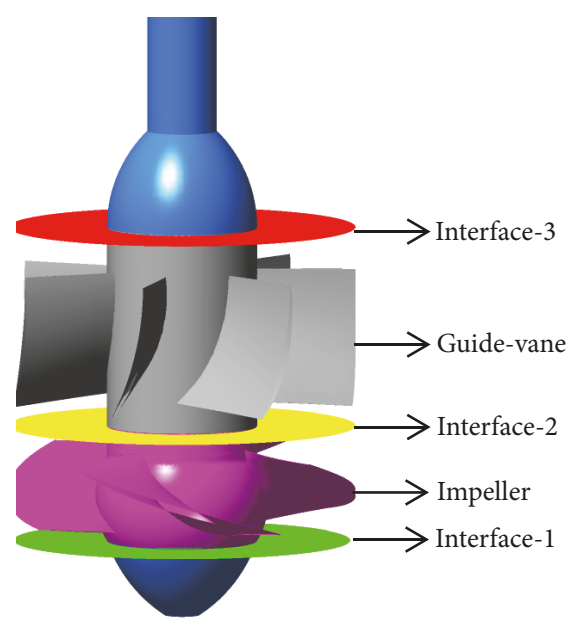

FIGURE 1: Pump model with 7 vanes.

reduction than leaned negative. Rodriguez [11] presents a theoretical analysis that allows us to describe the characteristics in the frequency domain of the vibration originated with the rotor-stator interaction in centrifugal pump turbine.

Although these investigations have helped us to deeply understand the nature of such rotor-stator-interaction, the vibroacoustics induced by rotor-stator interaction are seldom reported in the axial-flow pump. In this paper, we focused on the effect of guide vanes on flow and vibroacoustics in axial-flow pump. The N-S equations were simulated in axial-flow pump with four impeller blades, in which three diffuser models with 5, 7, and 9 vanes are devised to match respectively. A full scale structural vibroacoustics coupled model is solved using LMS acoustics software.

In what follows, the governing equations of the flow field will be explained in Section 2. Vibroacoustics induced by flow will then be described in Section 3. Conclusions close the paper in Section 4.

\section{Numerical Simulation}

The pump considered in this paper has four impeller blades, in which three diffuser models with 5, 7, and 9 vanes are devised to match, respectively, shown in Figure 1. The characteristic parameters of the pump are the rotor diameter $D_{2}=0.3 \mathrm{~m}$, flow rate at nominal operation condition $Q_{\mathrm{V}}=$ $1000 \mathrm{~m}^{3} / \mathrm{h}$, the corresponding pump head $H=6 \mathrm{~m}$, and rotation speed $n=1470 \mathrm{rpm}$.

2.1. Governing Equations. In present paper, the flow of fluid through the pump is considered as an incompressible fluid. Large eddy simulation (LES) method is applied to solve the filtered N-S equation to catch the detailed unsteady flow characteristics. LES focus on solving the large relevant turbulent scales, while small scales are removed from the simulated field with a low pass filter. The effect of the subgrid scales on the resolved scales is considered by an SGS model [12]. The governing equations are the spatially filtered continuity equation and the Navier-Stokes equations.

$$
\begin{gathered}
\frac{\partial \bar{u}_{i}}{\partial x_{i}}=0 \\
\frac{\partial \bar{u}_{i}}{\partial t}+\bar{u}_{j} \frac{\partial \bar{u}_{i}}{\partial x_{j}}=-\frac{1}{\rho} \frac{\partial \bar{p}}{\partial x_{i}}+\nu \frac{\partial^{2} \bar{u}_{i}}{\partial x_{j}^{2}}-\frac{\partial \tau_{i j}}{\partial x_{j}}
\end{gathered}
$$

where $\bar{u}_{i}$ is the grid-scale velocity component in the $x_{i}$ direction, $\bar{p}$ the grid-scale static pressure, $\rho$ the density, and $v$ the kinematic viscosity. Einstein's summation convention for repeated indices is used. $\tau_{\mathrm{ij}}$ is the subgrid-scale-stress tensor

$$
\tau_{i j}=\overline{u_{i} u_{j}}-\overline{u_{i}} \overline{u_{j}}
$$

In the present paper, the classical Smagorinsky model (Smagorinsky, 1963) is adopted, which assume the subgrid stress tensor to be proportional to the resolved rate of strain. Thus

$$
\tau_{i j}-\frac{\tau_{k k} \delta_{i j}}{3}=2 v_{t} \bar{S}_{i j}
$$

where $\bar{S}_{i j}$ is the filtered rate of strain tensor, given by

$$
\bar{S}_{i j}=\frac{\left(\partial \overline{u_{i}} / \partial x_{j}+\partial \overline{u_{j}} / \partial x_{i}\right)}{2}
$$

The eddy viscosity is expressed by

$$
v_{t}=C_{s}^{2} \Delta^{2} \sqrt{2 \bar{S}_{i j} \bar{S}_{i j}}
$$

where $C_{s}$ is Smagorinsky constant and $\triangle$ is filter width.

Central finite difference method is used to discretize the convection terms involved. Momentum and turbulent kinetic energy equations are discretized using second-order upwind scheme. The N-S equations are solved with the commercial code Ansys-Fluent. Second-order implicit format is used to discretize the time domain. SIMPLEC algorithm is adopted to calculate the pressure-velocity coupling. Before the unsteady flow solutions, steady flow is first computed and then used as initial condition to unsteady flow calculations. For the unsteady calculation, time step size is set to $1.13 \mathrm{e}-4 \mathrm{~s}$, which is equivalent to the time for impeller rotating one degree.

\subsection{Computational Domain and Boundary Conditions.}

Figure 2 describes the computational domain of pump model that is composed of inlet, rotor, stator outlet, and two identical right angle elbows. Moreover, the surface meshes of the rotor and stator are showed. Hexahedral elements are used and the blade zone is about 2.18 million cells, guide vanes zone about 1.37 million cells, the inlet and outlet zones about 537,000, and two elbows parts about 475,000 cells.

The boundary conditions imposed are a constant massflow rate at the inlet, outflow boundary condition at the outlet, and the no-slip conditions over wall. Before the unsteady flow solutions (LES), steady flow is first computed ( $\mathrm{k}-\varepsilon$ turbulence model), in which the turbulent intensity 


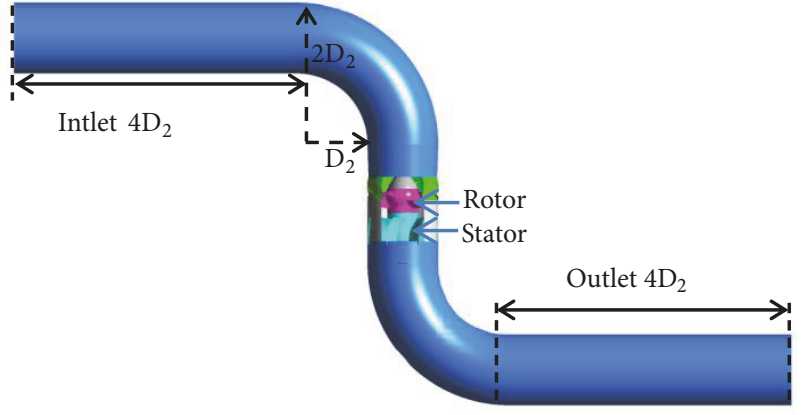

(a) 3D models of total domain including inlet, impeller blades, guide vanes, and outlet

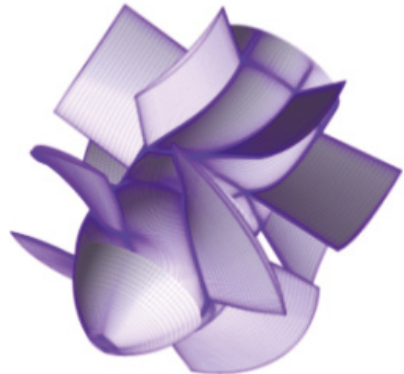

(b) Surface mesh of the rotor and stator

Figure 2: Pump model.

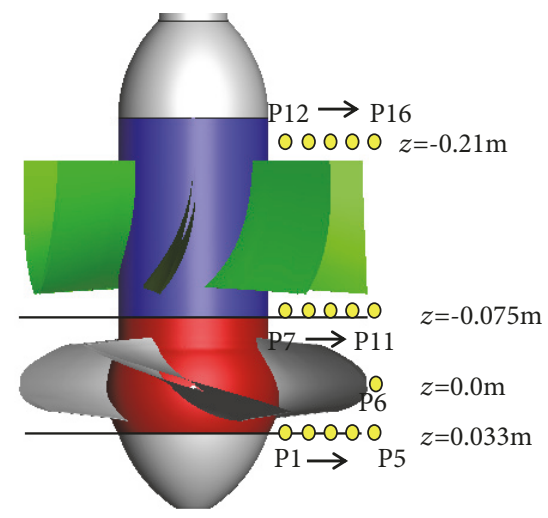

FIgURE 3: Location of monitor points.

is according to the formula $0.16 \mathrm{Re}^{-1 / 8}$ to different operating conditions and hydraulic diameter is $0.3 \mathrm{~m}$. Other turbulent model constants are $C_{\mu}=0.09, C_{1 \varepsilon}=1.42, C_{2 \varepsilon}=1.38$, $\sigma_{k}=0.72$, and $\sigma_{\varepsilon}=0.75$. Due to the rotation of impeller, two interfaces between the rotor and stator are formed, one of which is between the elbow and the impeller, and the other is between the impeller and guide vane.

2.3. Pressure Fluctuation. Figure 3 describes the monitoring points in the axial-flow pump, which is located on different sections along the axial direction, namely, the impeller inlet, tip clearance, the interface between the impeller and guide-vane, and guide-vane outlet (respectively, located on $z=0.033 \mathrm{~m}, z=0.0 \mathrm{~m}, z=-0.075 \mathrm{~m}$, and $z=-0.21 \mathrm{~m}$ ), where the points marked P5, P6, P11, and P16 are located at the inner surface of the casing, others at the inside of axial pump. In our previous studied work [13], the frequency spectrum of pressure fluctuations of monitoring points located at the inner surface of the casing was investigated. In this paper, we further evaluated the variation of pressure coefficient fluctuations along the radial direction.

Figure 4 represents the frequency spectrum characteristics of pressure coefficient fluctuations, defined as $C \mathrm{p}=2(p-\bar{p}) / \rho u_{2}^{2}$, at different cross sections inside the axial-flow pump with 7 vanes when operating at nominal flow rate, where $p$ and $\bar{p}$ are instantaneous pressure and timeaveraged pressure of the monitor points; $u_{2}$ represents the circumference speed of the impeller outlet. It can be seen that the pressure coefficient fluctuations, caused by impeller rotation, of monitoring points at impeller inlet are dominated by BPF and its harmonic frequency. The amplitude of pressure coefficient fluctuations gradually increases at first from hub to rim along the radial direction and then declines at the point P5 located on the same cross section of the inner surface of the casing as shown in Figure 5. For the vane inlet section $(z=-$ $0.075 \mathrm{~m}$ ), the amplitude of pressure coefficient fluctuations of monitoring points decreases significantly, which indicates that, after passing the impeller, pressure coefficient pulsation intensity becomes decreasing, and pressure coefficient fluctuations amplitude at low frequency becomes gradually visible. For the guide vanes outlet $(z=-0.21 \mathrm{~m})$, the amplitude of pressure coefficient pulsation of monitoring points continues to decrease. The pressure coefficient fluctuation of bladepassing frequency declines obviously and comparable to that of low frequency, which indicates that the effect of the rotating impeller on vane outlet pressure fluctuations becomes weak.

Figure 5 describes the frequency spectrum of pressure coefficient fluctuations of monitoring points located at the inner surface of the casing with 7 vanes when operating at nominal flow rate, from which it can be seen that the amplitude of pressure coefficient fluctuation is the largest on the point P6 located on the tip clearance, about 1.6 times of that at point P5 located on impeller inlet. The results indicate that nonuniform flow in the tip clearance may be a major contributor to large pressure fluctuation. After passing the impeller and the guide-vane, pressure coefficient declines gradually, consistent with that reflected by Figure 4 .

To verify the validity of computed results, pressure fluctuations in the axial-flow pump casing were measured at the experimental test system as shown in Figure 6. The test system mainly consists of storage water tank, inlet and outlet pipe, control valve, motor, axial-flow pump, high frequency sensor, and data acquisition system. Sample frequency is $25600 \mathrm{~Hz}$, sampling number is 65536, and sampling time is $2.56 \mathrm{~s}$. 


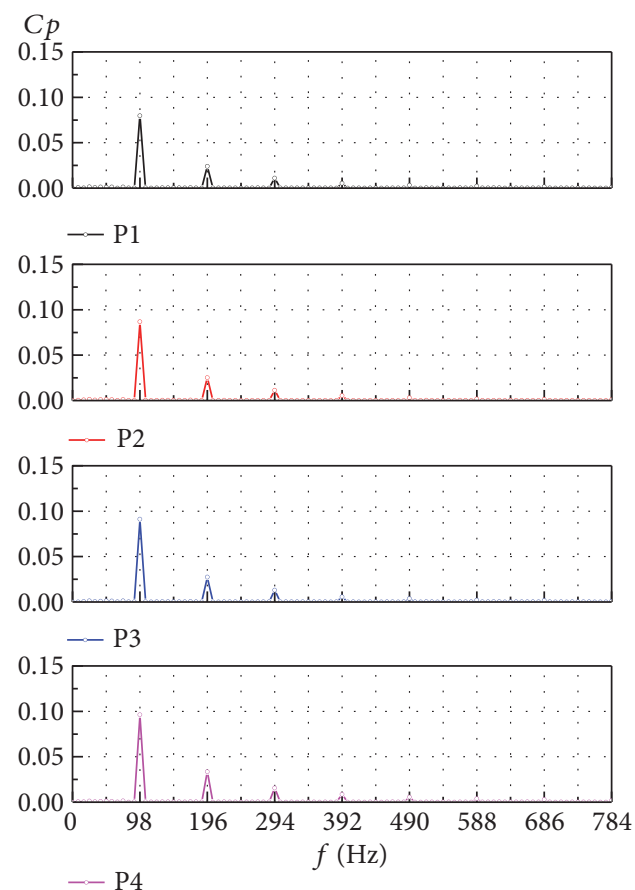

(a) $\mathrm{z}=0.033 \mathrm{~m}$
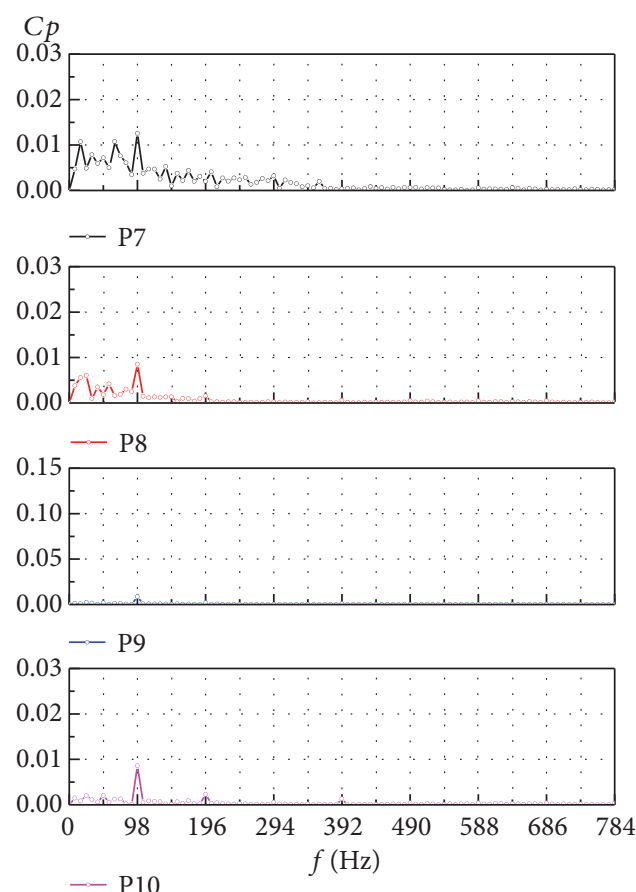

(b) $\mathrm{z}=-0.075 \mathrm{~m}$
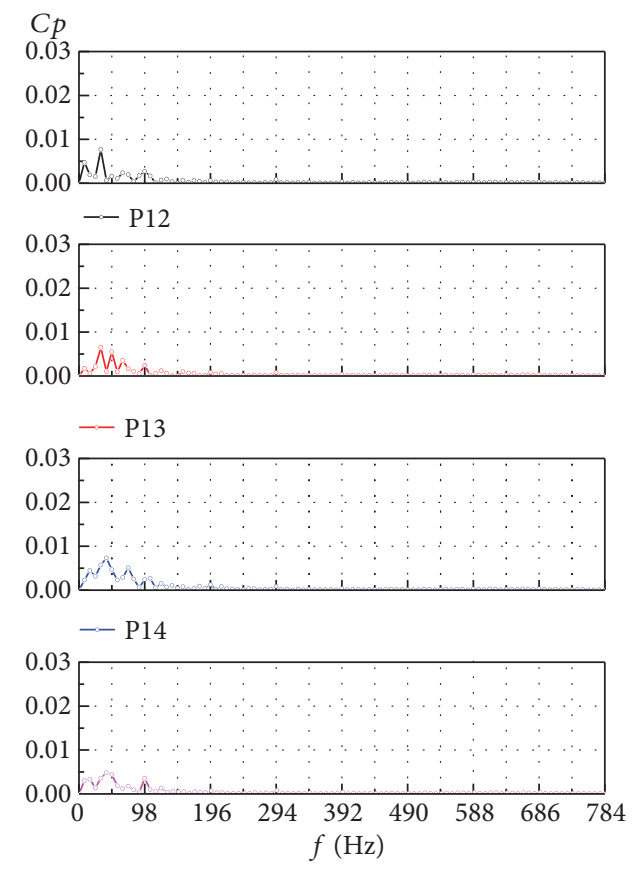

$\longrightarrow \mathrm{P} 15$

(c) $z=-0.21 \mathrm{~m}$

FIGURE 4: Frequency spectrums of pressure coefficient fluctuations. (a) Impeller inlet, (b) vane inlet, and (c) vane outlet.

Figure 7 shows the comparisons between the computed results and experimental test, where Figures $7(\mathrm{a})$ and $7(\mathrm{c})$ represent pressure coefficient fluctuation characteristics in time domain corresponding to point P5 and P6 shown in Figure 3, respectively; Figures 7(b) and 7(d) represent frequency spectrum of pressure coefficient corresponding to point P5 and P6. $\mathrm{t} / \mathrm{T}$ represents the ratio of computed time and rotor rotating period. These results indicated that pressure fluctuation is periodic and has four peaks and four valleys in one impeller rotation period, which is the same as blade number. The agreements between the numerical simulation and experimental values are relatively well, although small discrepancies do exist, which may be caused by different sampling frequency and sampling number.

Figure 8 describes the frequency spectrum of pressure coefficient fluctuations of monitoring point $\mathrm{P} 4$ with different 


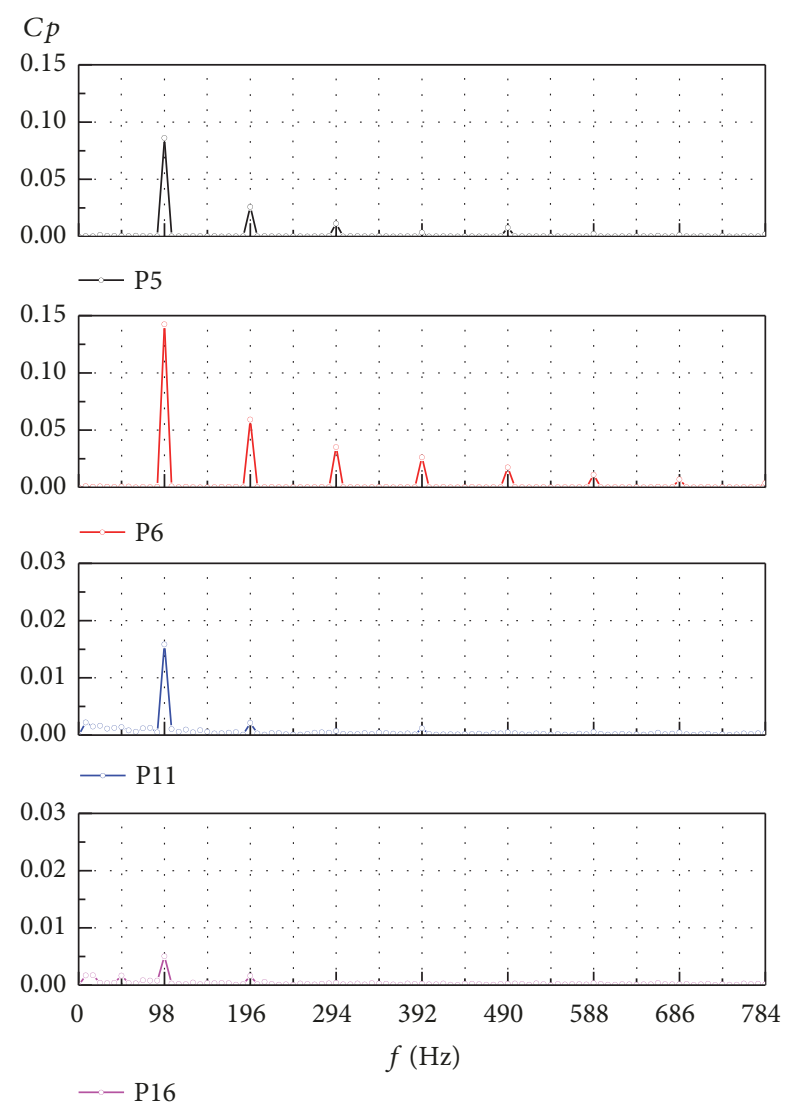

FIGURE 5: Frequency spectrum of pressure coefficient fluctuations.

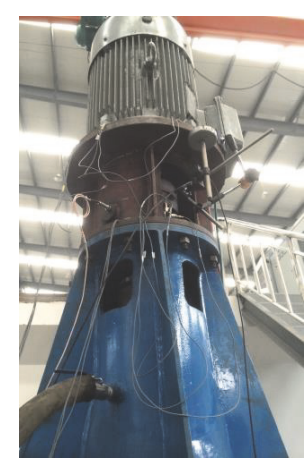

(a) The experimental test system

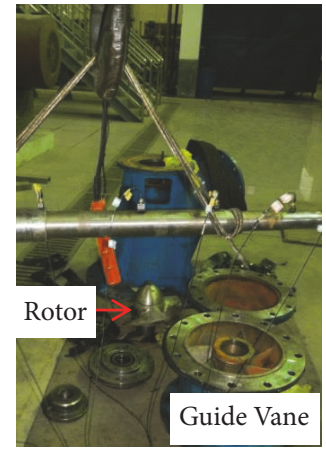

(b) The rotor and guide vane
FIGURE 6: Experimental test system.

vanes. It can be seen that the pressure coefficient fluctuations of the pump with 9-vane diffuser are smaller than other two cases at all working points, which implies that the reasonable increasing of the vane numbers of the diffuser is beneficial to control the pressure fluctuation in the axial-flow pumps. Moreover, the pressure coefficient fluctuating becomes strong as the flow discharge decreases rapidly from $1.0 Q_{\mathrm{v}}$ to $0.6 Q_{\mathrm{v}}$ in the pump matched three kinds of vane models, which indicates that the pump has strong exciting force as it is running at low discharge point. However, the pressure coefficient fluctuating also decreases slightly when the flow discharge increases from $1.0 Q_{v}$ to $1.3 Q_{v}$, which is contradiction with
TABLE 1: Coefficient of variance corresponding to four monitoring points.

\begin{tabular}{lccc}
\hline & $0.8 \mathrm{Qv}$ & $1.0 \mathrm{Qv}$ & $1.3 \mathrm{Qv}$ \\
\hline The max value at BPF & $37.97 \mathrm{kPa}$ & $24.67 \mathrm{kPa}$ & $25.35 \mathrm{kPa}$ \\
The min value at BPF & $33.84 \mathrm{kPa}$ & $22.75 \mathrm{kPa}$ & $22.91 \mathrm{kPa}$ \\
The mean value at BPF & $35.44 \mathrm{kPa}$ & $23.42 \mathrm{kPa}$ & $24.63 \mathrm{kPa}$ \\
The standard deviation & $1.77 \mathrm{kPa}$ & $0.86 \mathrm{kPa}$ & $1.16 \mathrm{kPa}$ \\
Coefficient of Variance & $4.9 \%$ & $3.6 \%$ & $4.7 \%$ \\
\hline
\end{tabular}

our knowledge that the minimum pressure fluctuating is associated with the pump operation around to best efficiency point. So we examine the influence of the flow nonuniformity induced by the suction elbow on the pump circumferential unsteady behavior at operator point. Four monitoring points, P5, P5-1, P5-2, and P5-13, are located on the impeller inlet $(z=0.033 \mathrm{~m})$ as shown in Figure 9(a). Figures 9(b) $-9(\mathrm{~g})$ describe the pressure fluctuations and frequency spectrum of four monitoring point with 7-vanes at $0.8 Q_{\mathrm{v}}, 1.0 \mathrm{Q}_{\mathrm{v}}$, and 1.3 $Q_{\mathrm{v}}$, respectively. It can be seen that the amplitude of pressure fluctuation exists in circumferential nonuniformity due to the flow nonuniformity induced by the suction elbow. Table 1 presents the coefficient of variance at BPF corresponding to four monitoring points, from which it can be seen that the maximum value of pressure fluctuations are $37.97 \mathrm{kPa}$, $24.67 \mathrm{kPa}$, and $25.35 \mathrm{kPa}$ to the axial-flow pump with 7 vanes at different operation point, respectively, the mean values are $35.44 \mathrm{kPa}, 23.42 \mathrm{kPa}$, and $24.63 \mathrm{kPa}$, respectively, and the coefficient of variance is $4.9 \%, 3.6 \%$, and $4.7 \%$, respectively, which indicates that the circumferential unsteady behavior of pressure fluctuation is more severe due to flow nonuniformity induced by the suction elbow at partial operation. Generally, the pressure fluctuating increases slightly when the flow discharge increases from $1.0 Q_{\mathrm{v}}$ to $1.3 Q_{\mathrm{v}}$.

\section{Vibration and Noise Simulation}

Unsteady flow exciting forces acting on the axial-flow pump give rise to structural vibration and further radiate noise to the environment. In the present study, the interaction process among the fluid-structure-acoustic is simulated by coupling way as follows: (1) LES method is used to solve the 3D unsteady flow, which supplies a time series for the pressure fluctuations at the interface between the fluid and wall, while the feedback effects of structural vibration on the fluid are neglected. (2) Finite element method is used to simulate structural vibration induced by the unsteady flow, and the noise field generated by the vibration is solved using boundary element method. The mutual coupling interaction between the vibration and noise field in the fluid is considered using the vibroacoustic coupling model, which supplies the vibration velocities on the outer surface of pump. (3) Noise field radiated to the environment is solved using boundary integral equation method.

3.1. Acoustic Model. The governing equation for acoustic fields generated by the vibration is Helmholtz equation:

$$
\nabla^{2} p+k^{2} p=0
$$




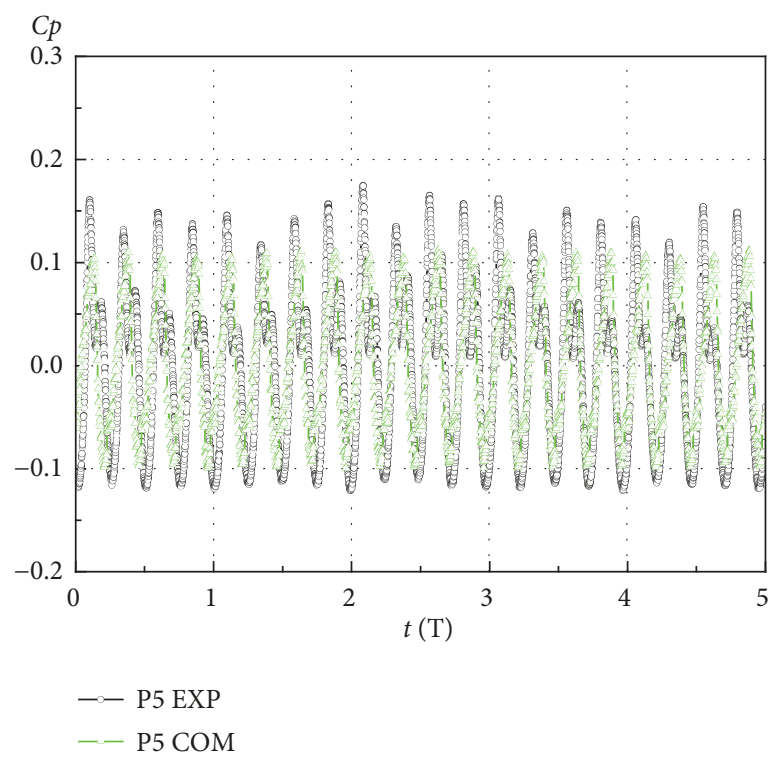

(a) Pressure fluctuation characteristics in time domain

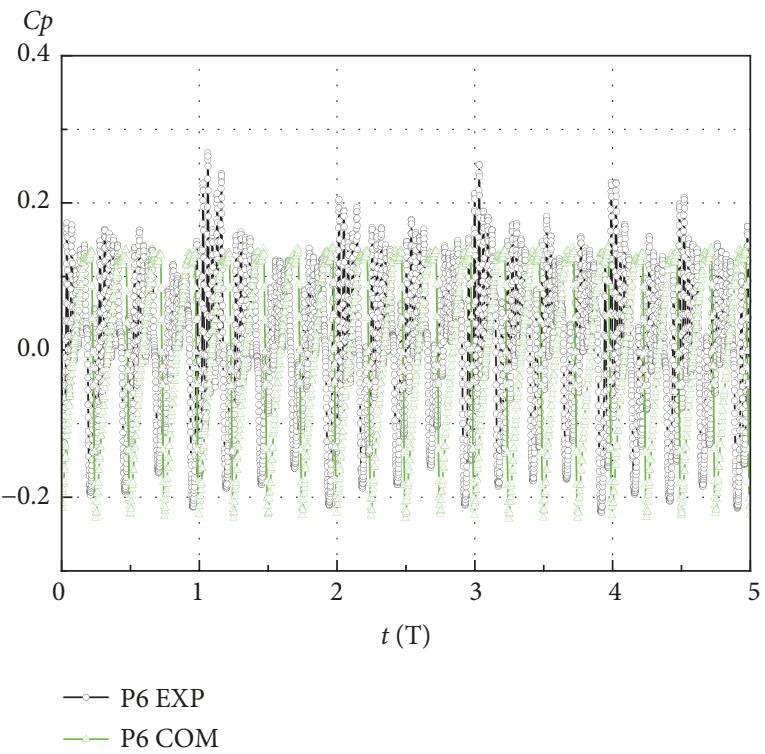

(c) Pressure fluctuation characteristics in time domain

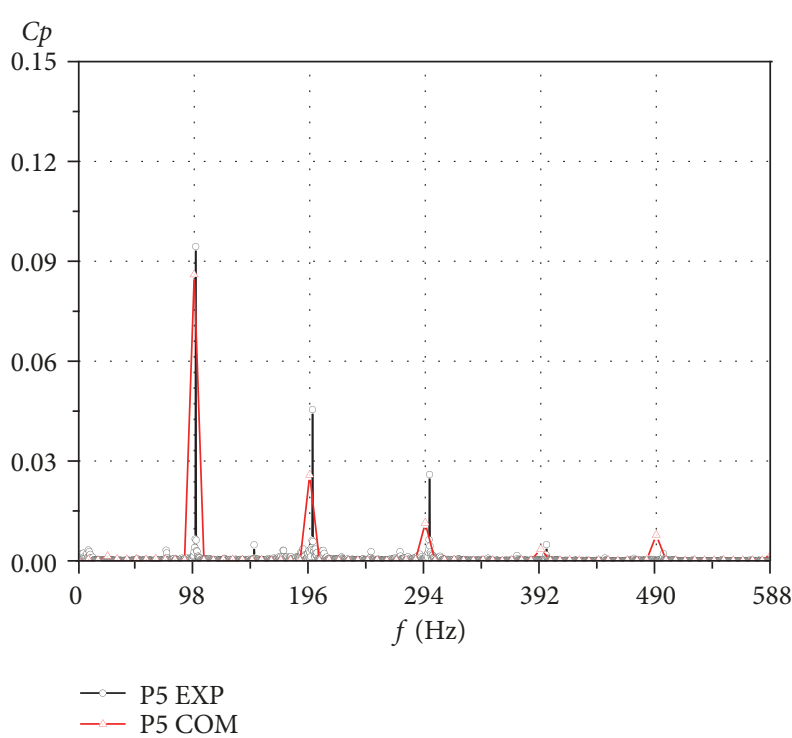

(b) Frequency spectrum of pressure coefficient fluctuations

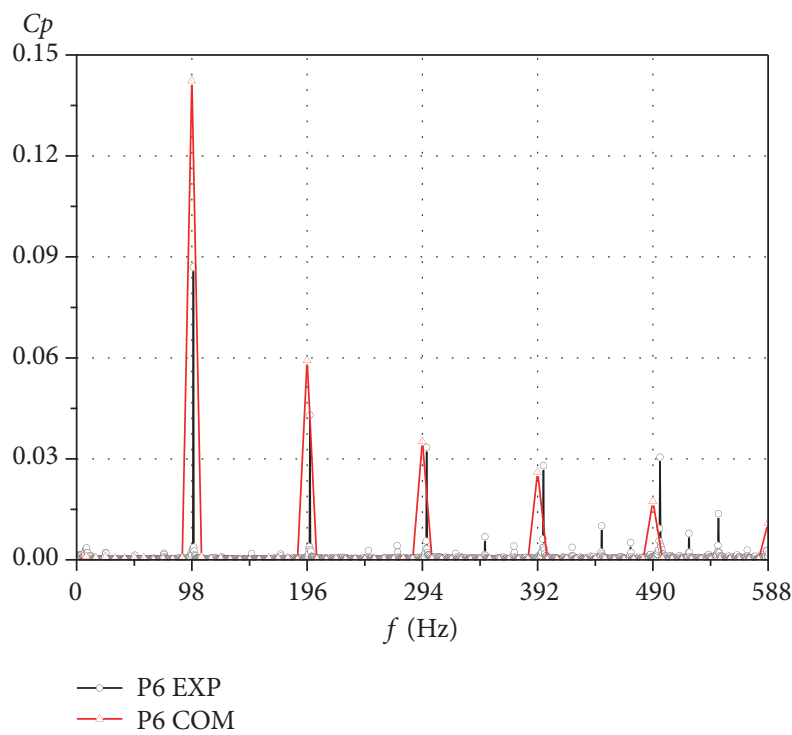

(d) Frequency spectrum of pressure coefficient fluctuations

FigURE 7: Comparison of pressure coefficient fluctuations.

where $p$ is the sound pressure amplitude, $k=\omega / c$ the wavenumber, $\omega$ the angular frequency, and $c$ the sound speed.

In the present study, (6) is solved using boundary integral equation method, which has been widely used in acoustics. The boundary integral formulation equation for an interior acoustic problem corresponding to (6) is

$$
C(\vec{r}) p(\vec{r})=\int_{S}\left(p\left(\vec{r}_{a}\right) \frac{\partial G}{\partial n}+j \rho_{0} \omega v_{n}\left(\vec{r}_{a}\right) G\right) \mathrm{d} S
$$

where $p\left(\vec{r}_{\mathrm{a}}\right)$ and $v_{\mathrm{n}}\left(\overrightarrow{\mathrm{r}}_{\mathrm{a}}\right)$ are the sound pressure and the particle normal velocity on the boundary surface $S$, respectively, $\rho_{0}$ mean fluid density, and $G$ the Green kernel func tion,

$$
G\left(\vec{r}, \vec{r}_{a}\right)=\frac{e^{-j k\left|\vec{r}-\vec{r}_{a}\right|}}{4 \pi\left|\vec{r}-\vec{r}_{a}\right|}
$$

The leading coefficient $\mathrm{C}(\overrightarrow{\mathrm{r}})$ is equal to 1 for $\overrightarrow{\mathrm{r}}$ in the domain and $1 / 2$ for $\vec{r}$ on a smooth boundary, and it is equal to 0 for $\vec{r}$ in the exterior domain.

To solve the Helmholtz integral equation numerically, (7) is first discretized and evaluated for each node $i$ on the boundary. This produces

$$
[\mathrm{A}]\left\{p_{i}\right\}=j \rho_{0} \omega[\mathrm{B}]\left\{V_{n i}\right\}
$$

where $\mathrm{A}$ and $\mathrm{B}$ denote global coefficient matrix. For the each node $b$, this produces

$$
A_{b i}=\delta_{b i}\left[1+\frac{1}{4 \pi} \int_{S} \frac{\partial}{\partial n}\left(\frac{1}{\left|\vec{r}_{b}-\vec{r}_{a}\right|}\right) \mathrm{d} S\right]
$$




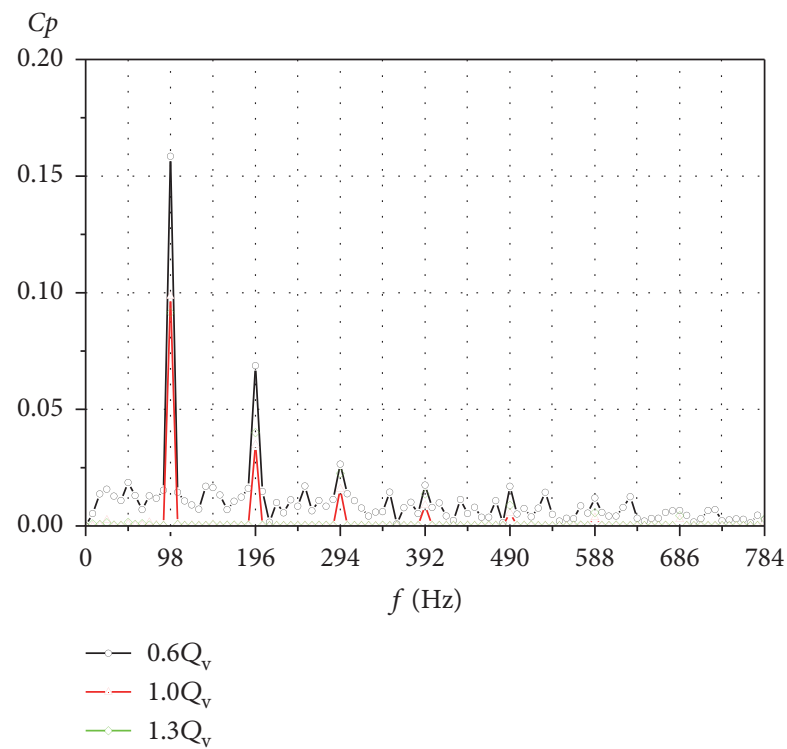

(a) 5 vanes

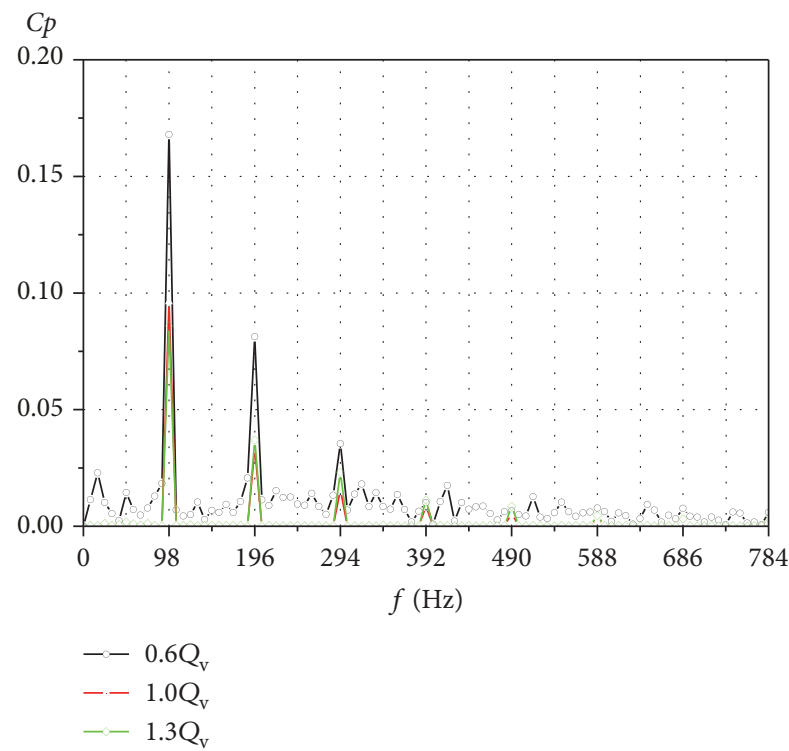

(b) 7 vanes

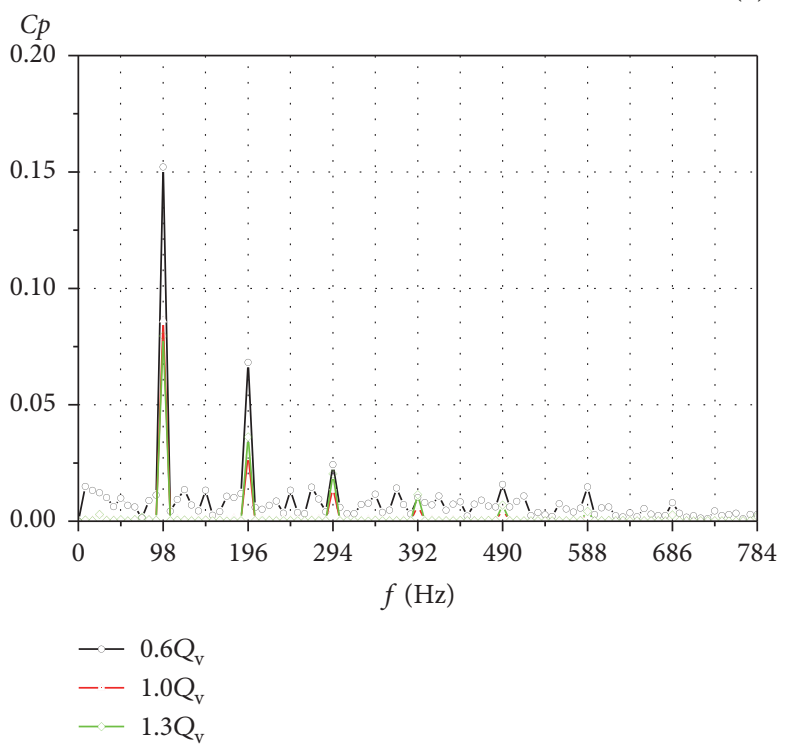

(c) 9 vanes

FIGURE 8: Frequency spectrums of pressure coefficient fluctuations at different flow discharge.

$$
\begin{array}{r}
-\int_{S} N_{i} \frac{\partial G}{\partial n} \mathrm{~d} S \\
B_{b i}=\int_{S} N_{i} G \mathrm{~d} S
\end{array}
$$

where $\delta_{\text {bi }}$ represents the Kronecker delta function and $N_{\mathrm{i}}$ the shape function.

3.2. Vibration Model. The finite element model of structure vibrations yields

$$
\left([\mathrm{K}]+j \omega[\mathrm{C}]-\omega^{2}[\mathrm{M}]\right)\left\{u_{i}\right\}+\left[\mathrm{L}_{c}\right]\left\{p_{i}\right\}=\left\{F_{s}\right\}
$$

where $\left\{\mathrm{u}_{\mathrm{i}}\right\}$ denotes a displacement vector, $[\mathrm{K}]$ stiffness matrices, $[\mathrm{C}]$ damping matrices, and $[\mathrm{M}]$ mass matrices. $\left[\mathrm{L}_{\mathrm{c}}\right]\left\{\mathrm{p}_{\mathrm{i}}\right\}$ is the force loading of the acoustic pressure on the structure surface. The symbol $\left[\mathrm{L}_{\mathrm{c}}\right]$ denotes fluid-structural coupling matrix, which is given by

$$
\mathrm{L}_{c}=-\sum_{e=1}^{m} \int_{S_{e}}\left(\left[N_{s}\right]^{\mathrm{T}}\right)\left\{n^{e}\right\}\left[N_{a}\right] \mathrm{d} S
$$

where $m$ denotes the coupling-surface element numbers. $N_{s}$ and $N_{\mathrm{a}}$ represent the structure element shape function and acoustic element shape function, respectively. $n^{\mathrm{e}}$ represents the unit normal vector. $\left\{F_{s}\right\}$ represents the flow excitation force, yielding

$$
F_{s}=\sum_{e=1}^{m} \int_{S_{e}}\left(\left[N_{s}\right]^{\mathrm{T}}\right)\left\{n^{e}\right\} \bar{p} \mathrm{~d} S
$$




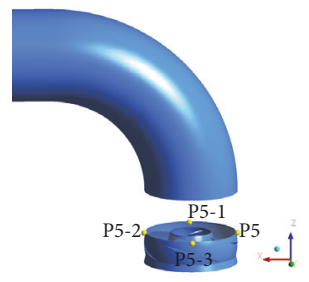

(a) Location of monitor points

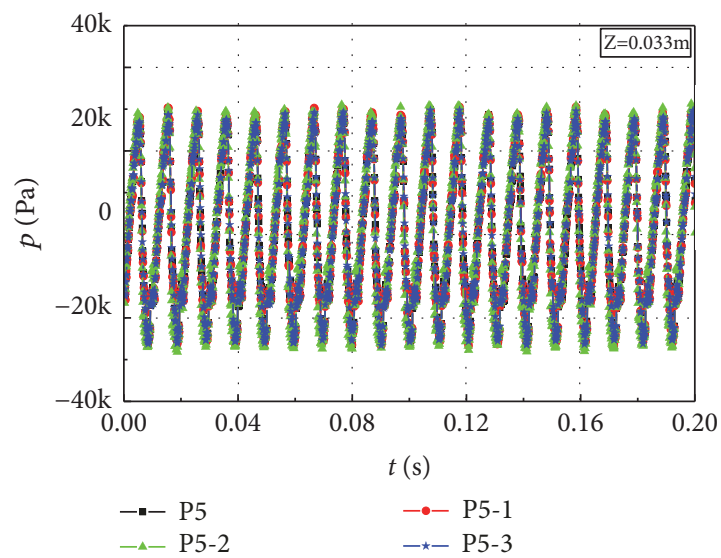

(d) Pressure fluctuation characteristics in time domain at $1.0 \mathrm{Qv}$

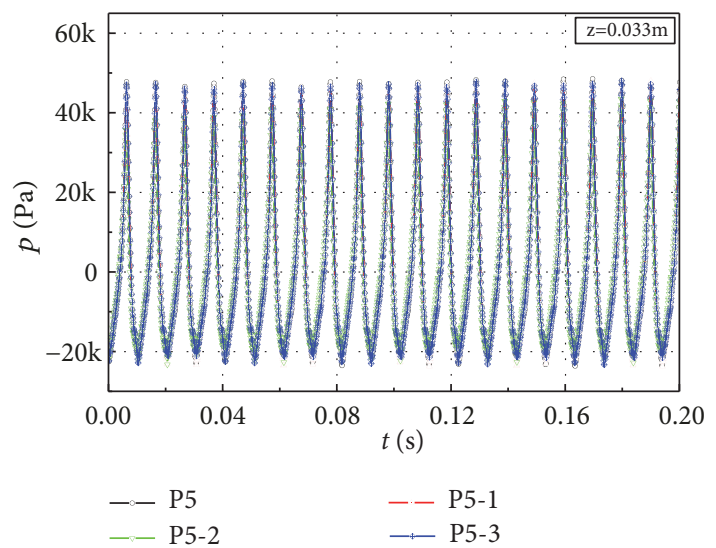

(f) Pressure fluctuation characteristics in time domain at $1.3 \mathrm{Qv}$

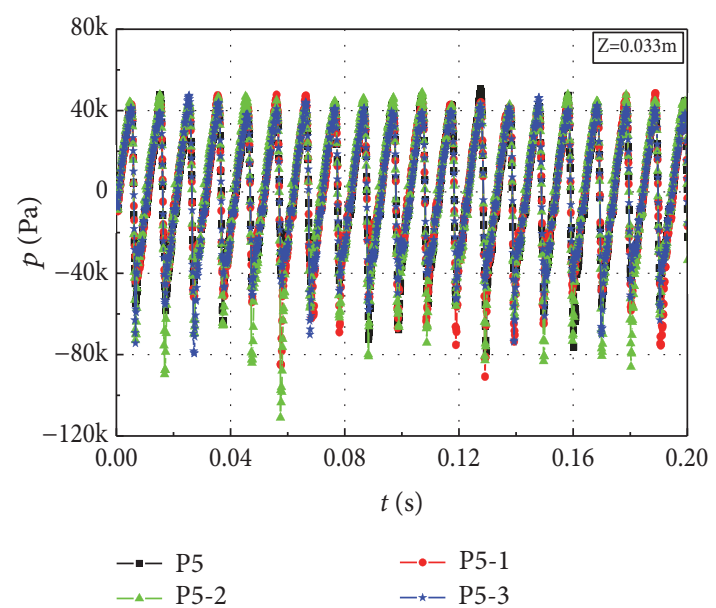

(b) Pressure fluctuation characteristics in time domain at $0.8 \mathrm{Qv}$

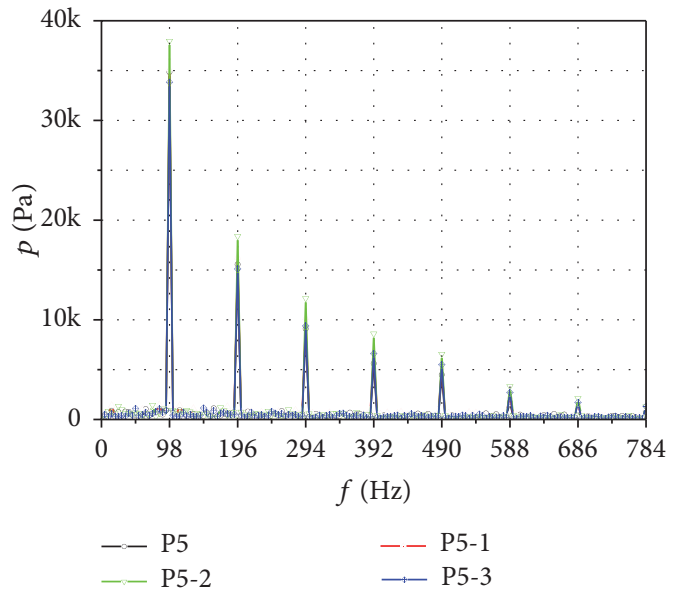

(c) Frequency spectral of pressure fluctuation at $0.8 \mathrm{Qv}$

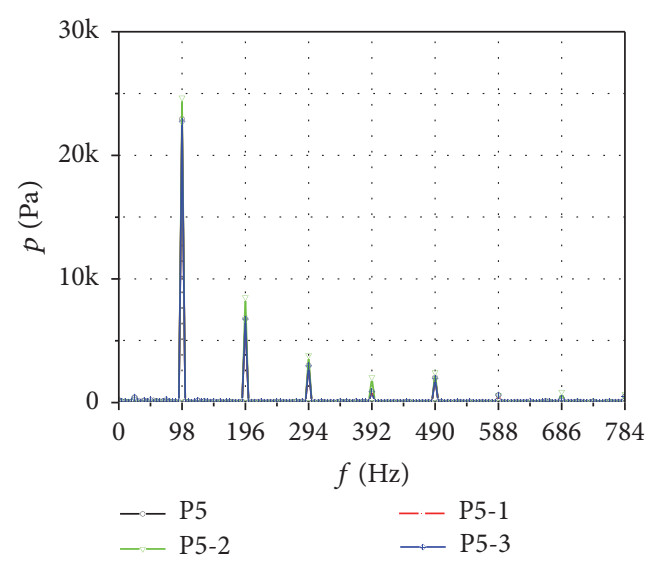

(e) Frequency spectral of pressure fluctuation at $1.0 \mathrm{Qv}$

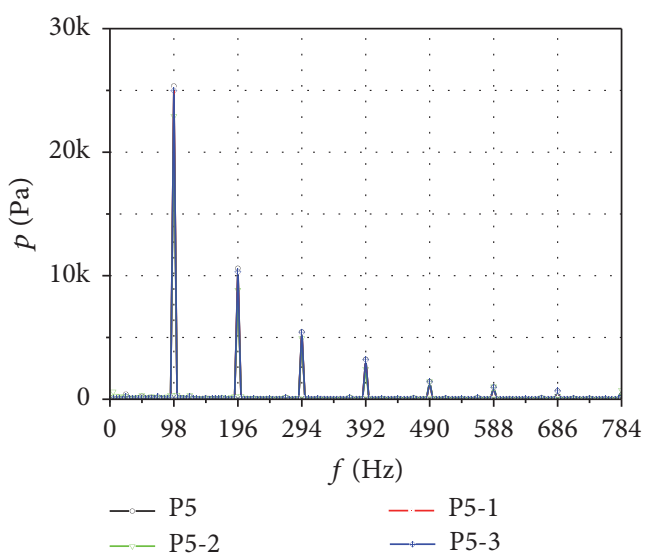

(g) Frequency spectral of pressure fluctuation at $1.3 \mathrm{Qv}$

Figure 9: Pressure fluctuations amplitude at BPF with 7 vanes.

In the present paper, the mutual interaction of fluidstructure is simulated by one-way weak coupling, in which the wall vibration is negligible.

For coupled vibroacoustic problems, an acoustic and a structural problem must be solved simultaneously to include the mutual coupling interaction between the fluid pressure and the structural deformation. The normal fluid velocity, $V_{\mathrm{ni}}$, must equal the normal structural velocity along the fluid-structure coupling interface,

$$
\left\{V_{n i}\right\}=j \omega[\mathrm{T}]\left\{u_{i}\right\}
$$

where $[T]$ is the transformation matrix.

The nonreflecting condition is carried out at the inlet and outlet. According to linear time-harmonic acoustic simulation rule, six linear elements per wavelength are required. 


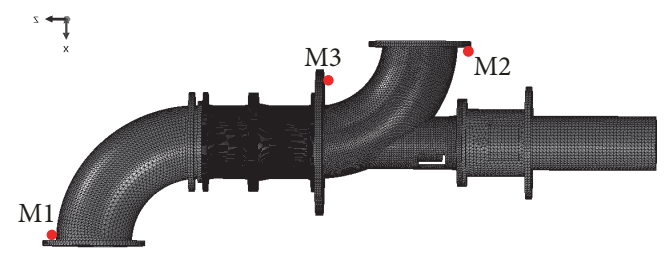

FIGURE 10: Mesh used in structural simulation.
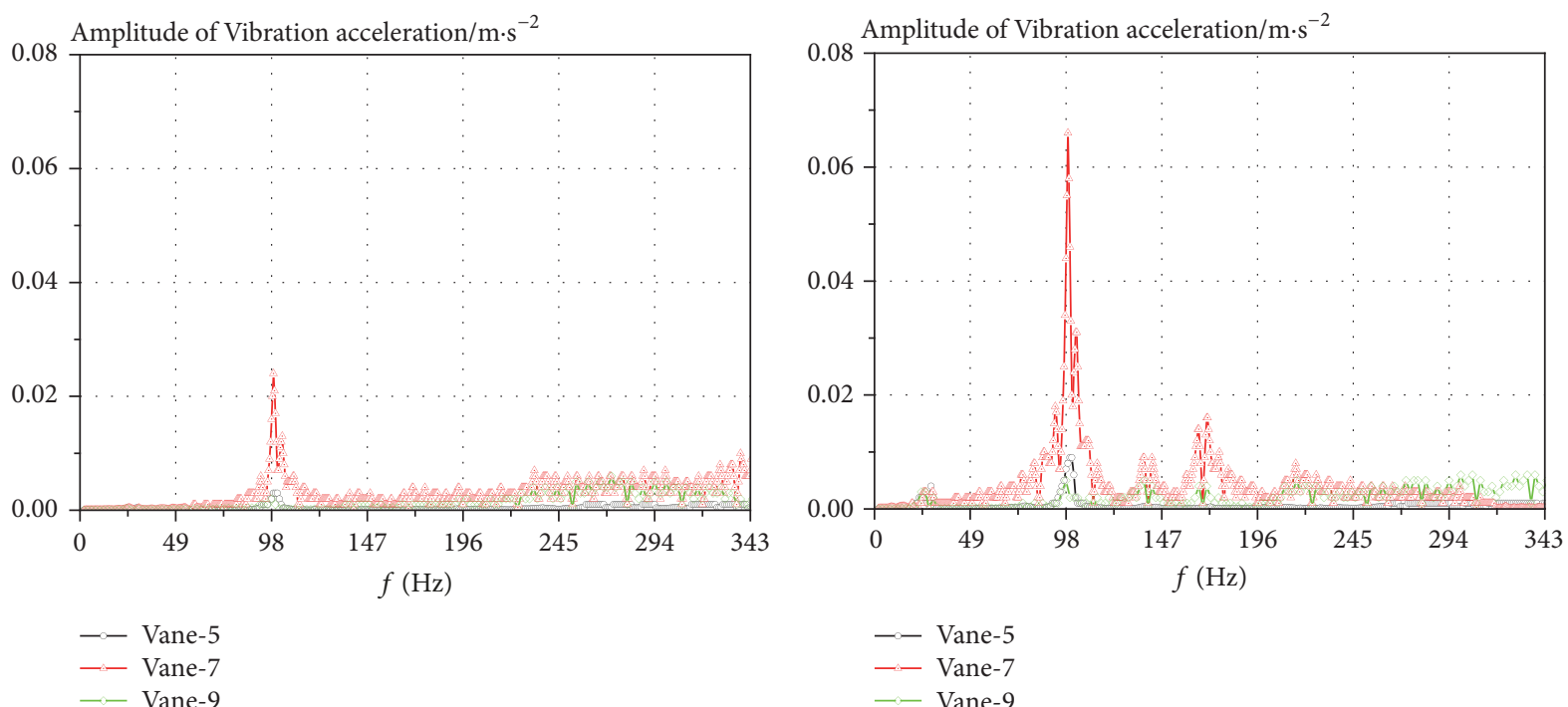

(a) M1

(b) M2

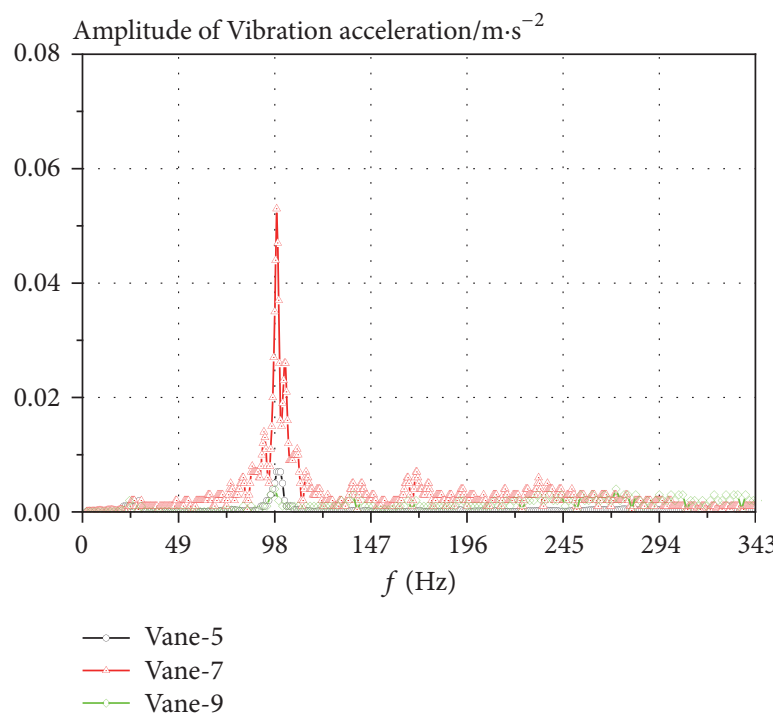

(c) $\mathrm{M} 3$

FIGURE 11: Frequency spectrums of vibration acceleration.

3.3. Numerical Results. Full scale axial pump structural vibroacoustics are simulated by LMS Virtual Lab software. Figure 10 shows a full scale structure mesh, which has 0.58 million 4-noded tetrahedral elements. The coefficients used are Elasticity modulus $E=210 \mathrm{GPa}$, density $\rho=7850 \mathrm{Kg} / \mathrm{m}^{3}$, and Poisson ratio $\sigma=0.3$. Moreover, for validating computed modal, the comparisons between the computed results and experimental results are performed, a detailed description of which can be seen in [13].

Figure 11 shows the vibration acceleration frequency spectrum characteristics for sampled points M1, M2, and M3, shown in Figure 10, when the axial-flow pump works 


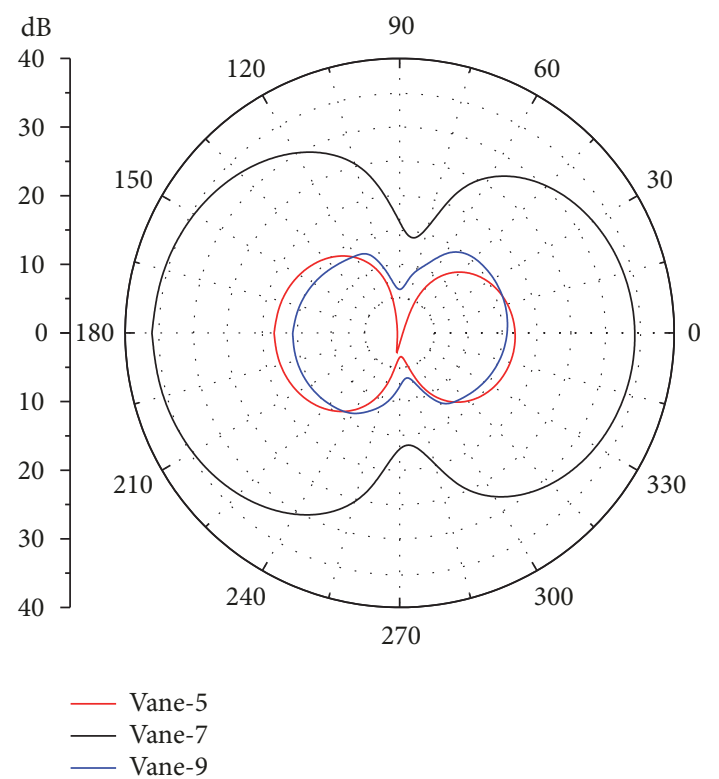

FIGURE 12: Monitoring points position and directivity distribution of radiation noise field. Directivity distribution $(f=98 \mathrm{~Hz})$.

at nominal flow rate. These sampled points are located on the inlet and outlet flange and the pump seat, respectively. It can be seen that the structural vibration is dominated by the BPF component, which is in accordance with frequency spectral characteristics of pressure pulsation. The amplitude of vibration acceleration with 7-vane diffuser is bigger than other pumps and with 9-vane diffuser is small relatively. This trend coincides with the law of exciting force as mentioned.

Figure 12 shows acoustics field directivity distribution of thirty-sixth monitoring points, located at 1.15 meter away from the center of the rotor, induced by the vibration of axialflow pump with different vanes corresponding to BPF, from which it can be seen that the noise field directivity distributions are typical dipoles. This is conforming to the fact that only the dynamic surface pressure (dipole source) is loaded and the monopole and quadrupole contributions of fluidacoustic sources are neglected in the coupled simulation.

The sound pressure level (SPL $=20 \times \log (p / 2 e-5))$ of radiation noise field excited by the pump vibration reaches the maximum value at the pump outlet direction either to the 5 -vanes or to 7 -vanes and 9-vanes, that is, about $18.3 \mathrm{~dB}$, $36.1 \mathrm{~dB}$, and $15.6 \mathrm{~dB}$, respectively, from which it can be seen that radiation noise field excited by the pump vibration with 9-vane diffuser is small relative to other two models for BPF components, which indicated that appropriate guide-vane numbers contribute to suppressing pressure fluctuations and vibroacoustics in axial-flow pump.

\section{Conclusions}

This study presented an effect of guide-vane numbers on pressure fluctuations and structural vibroacoustics induced by unsteady flow. The vibroacoustic field of the axial-flow pump is simulated using coupled vibroacoustic model. Some conclusions as follows:
(1) The dominant frequency of pressure fluctuations is the blade-passing frequency and its harmonic frequency, and the amplitude at the tip clearance is prevailing. The unsteady pressure fluctuating becomes strong as the flow discharge decreases from $1.0 Q_{\mathrm{v}}$ to $0.6 Q_{\mathrm{v}}$, the circumferential unsteady behavior of which is more severe due to flow nonuniformity induced by the suction elbow at partial operation. Generally, the pressure fluctuating increases slightly when the flow discharge increases from $1.0 Q_{v}$ to $1.3 Q_{v}$. Pressure fluctuations amplitude on the pump with 9-vane diffuser is small relative to other two models

(2) The dominant frequency of the vibration acceleration of axial-flow pump is also blade-passing frequency, which is consistent with pressure fluctuation frequency spectral characteristics. The vibrating accelerating and radiation sound field on the pump with 9-vane diffuser are also slight relatively at BPF, which indicated that appropriate guide-vane numbers contribute to suppress pressure fluctuations and vibration in axial-flow pump.

\section{Data Availability}

The data used to support the findings of this study are available from the corresponding author upon request.

\section{Conflicts of Interest}

The authors declare that there are no conflicts of interest regarding the publication of this paper.

\section{Acknowledgments}

This work was supported by the National Natural Science Foundation of China (51106099 and 11502146), the Natural Science Foundation of Shanghai (15ZR1429600), and Research Fund of Science and Technology Commission of Shanghai Municipality (13DZ2260900).

\section{References}

[1] D. Kaya, "Experimental study on regaining the tangential velocity energy of axial flow pump," Energy Conversion and Management, vol. 44, no. 11, pp. 1817-1829, 2003.

[2] W. D. Shi, S. Q. Wu, and J. Yao, "Rotor/stator interactions study under different operating condition in axial flow pump," Applied Mechanics and Materials, vol. 456, pp. 168-172, 2014.

[3] N. Arndt, A. J. Acosta, C. E. Brennen, and T. K. Caughey, "RotorStator Interaction in a Diffuser Pump," Journal of Turbomachinery, vol. 111, no. 3, pp. 213-221, 1989.

[4] C. Kang, X. Yu, W. Gong, C. Li, and Q. Huang, "Influence of stator vane number on performance of the axial-flow pump," Journal of Mechanical Science and Technology, vol. 29, no. 5, pp. 2025-2034, 2015.

[5] H. Wang and H. Tsukamoto, "Fundamental analysis on rotorstator interaction in a diffuser pump by vortex method," Journal of Fluids Engineering, vol. 123, no. 4, pp. 737-747, 2001.

[6] H. Zhang, W.-D. Shi, B. Chen, Q.-H. Zhang, and W.-D. Cao, "Experimental study of flow field in interference area between impeller and guide vane of axial flow pump," Journal of Hydrodynamics, vol. 26, no. 6, pp. 894-901, 2015. 
[7] W. C. Zierke, K. J. Farrell, and W. A. Straka, "Measurements of the tip clearance flow for a high-Reynolds-number axial flow rotor," Journal of Turbomachinery, vol. 117, no. 4, pp. 522-532, 1995.

[8] W. C. Zierke, W. A. Straka, and P. D. Taylor, "Experimental investigation of the flow through an axial-flow pump," Journal of Fluids Engineering, vol. 117, no. 3, pp. 485-490, 1995.

[9] A. Javadi, A. Bosioc, H. Nilsson, S. Muntean, and R. SusanResiga, "Experimental and numerical investigation of the precessing helical vortex in a conical diffuser, with rotor-stator interaction," Journal of Fluids Engineering, vol. 138, no. 8, pp. 113, 2016

[10] H. Liu, H. Ouyang, Y. Wu, J. Tian, and Z. Du, "An analytical investigation of stator lean on rotor-stator interaction noise," Journal of Aerospace Engineering, vol. 229, no. 1, pp. 96-109, 2015.

[11] C. G. Rodriguez, E. Egusquiza, and I. F. Santos, "Frequencies in the vibration induced by the rotor stator interaction in a centrifugal pump turbine," Journal of Fluids Engineering, vol. 129, no. 11, pp. 1428-1435, 2007.

[12] J. Meyers, Accuracy of Large-Eddy simulation strategies [Ph.D. thesis], Katholieke Universiteit Leuven, Belgium, 2004.

[13] E. Chen, Z. Ma, G. Zhao, G. Li, A. Yang, and G. Nan, "Numerical investigation on vibration and noise induced by unsteady flow in an axial-flow pump," Journal of Mechanical Science and Technology, vol. 30, no. 12, pp. 5397-5404, 2016. 


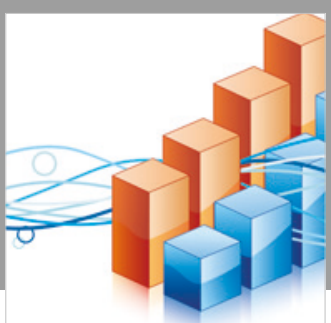

Advances in

Operations Research

\section{-n-m}
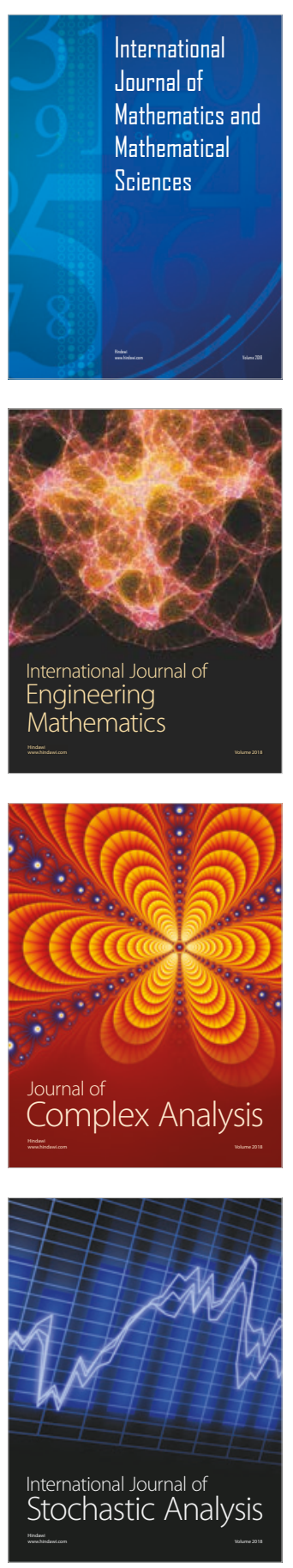
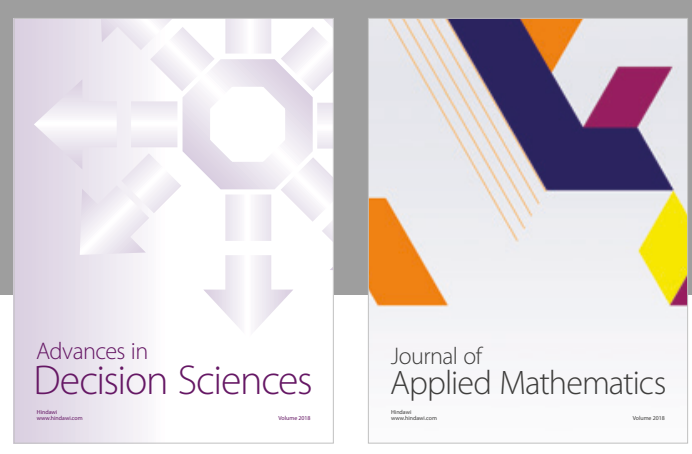

Journal of

Applied Mathematics
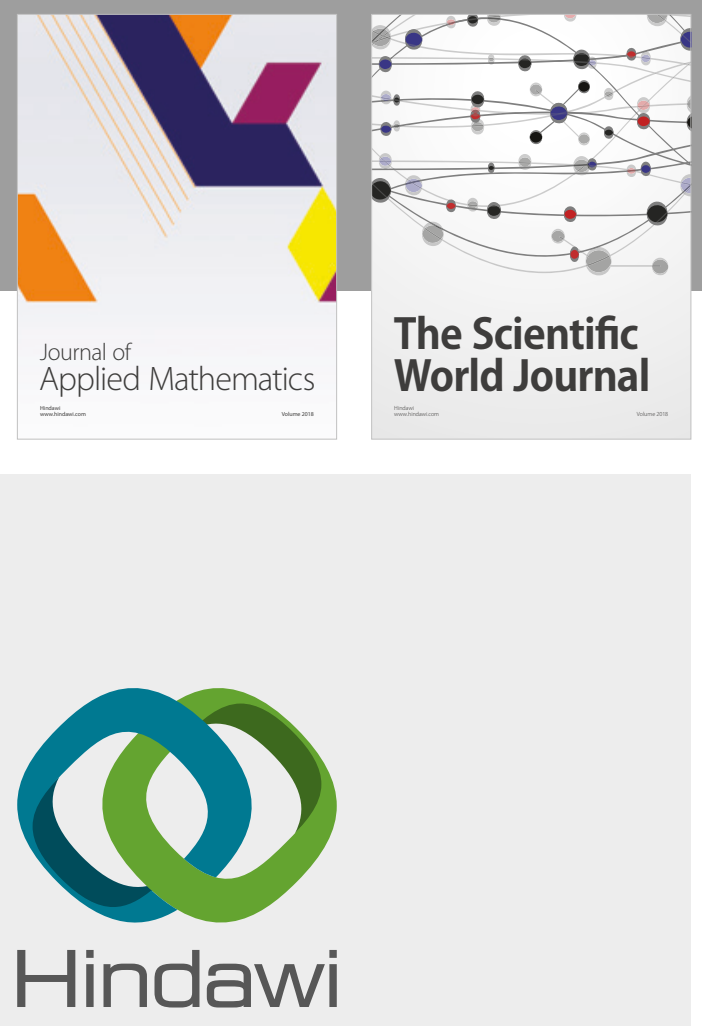

Submit your manuscripts at

www.hindawi.com

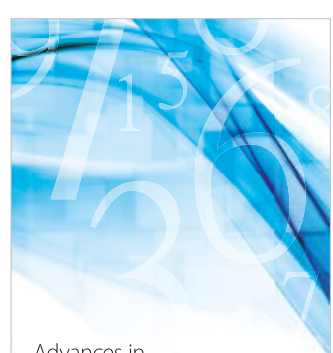

Advances in
Numerical Analysis
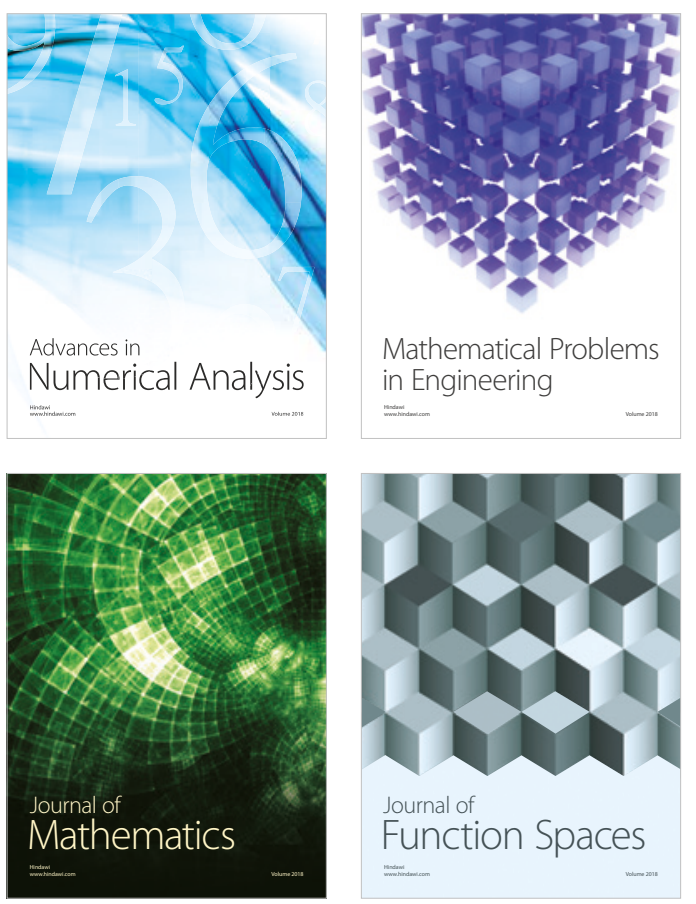

Mathematical Problems in Engineering

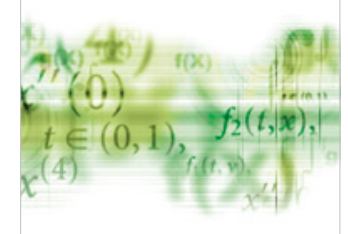

International Journal of

Differential Equations

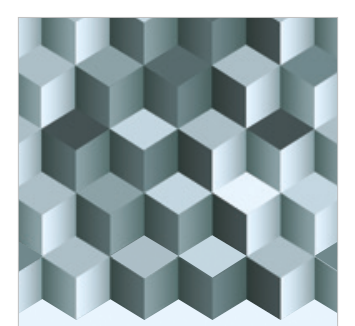

Journal of

Function Spaces

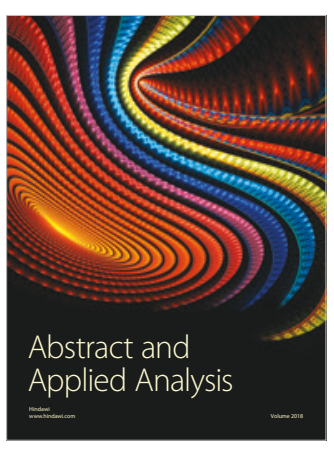

The Scientific

World Journal

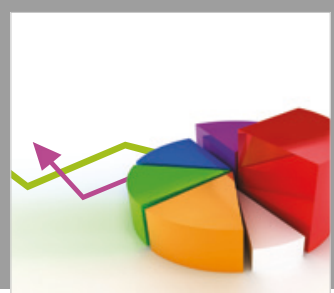

Journal of

Probability and Statistics
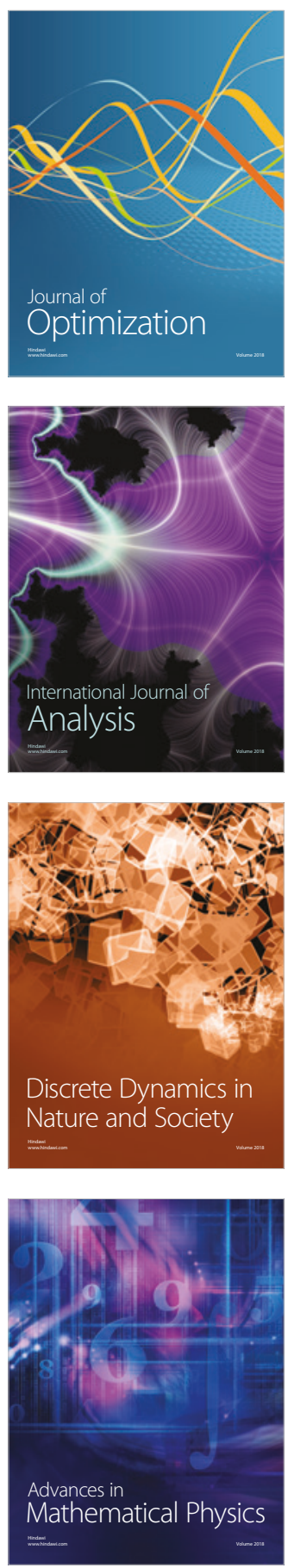